\title{
Use of Inertial Central to Analyse Skill of Inter-Limb Coordination in Sport Activities
}

\author{
Ludovic Seifert1, Maxime L'Hermette1, Léo Wattebled1, John Komar1, Florian Mell1, \\ David Gomez2, Yanis Caritu2 \\ 1 CETAPS - EA 3832, Faculty of Sport Sciences, University of Rouen, France \\ 2 MOVEA-Gyration, Grenoble, France \\ Ludovic.seifert@univ-rouen.fr
}

\begin{abstract}
Kinematical movement analysis is regularly conducted through $3 D$ video device, studying great number of parameters. The aim of this study is to focus on movement variability, following dynamical system perspectives, that is suitable through the use of inertial central to collect data in ecological context. This study gives two examples of sport activity (breaststroke swimming and rock climbing) for which the motor skill was analysed through a macroscopic parameter, notably upper-lower limb coordination. The results showed higher intraindividual movement variability in experts while beginners used basic modes of coordination.
\end{abstract}

\section{Introduction}

The main goal of the dynamical system theory of motor control is to study the change between steady states of the system through time, particularly by focusing on variability and stability of the order parameter (i.e. specific parameter that qualitatively captures the state of system). In the human motor control during sport activity area, the temporal dynamic of inter-limb coordination is analysed by identifying the attractors (steady states) and the bifurcation between attractors [1].

In that way, 3D kinematical movement analysis was regularly conducted using numeric video or infra-red camera. However, the use of a system with multiple cameras (i) is time consuming, (ii) prevents analysis in an ecological context, and (iii) prevents analysis of long time-series. The use of inertial central looks like an accurate and reliable device to analyse movement variability from a macroscopic approach; notably in order to explore the role played by intra-individual variability in skill motor behaviour.

\section{Methods}

Four inertial central MotionPod3 (Movéa, Grenoble) composed by 3D accelerometer, 3D gyroscope and 3D magnetometer enable to analyse (i) absolute values of roll, pitch and yaw of each sensor with a local reference (i.e. egocentric) or allocentric (north, east, gravity, respectively for the $\mathrm{x}, \mathrm{y}, \mathrm{z}$ axis), and (ii) relative values of roll, pitch and yaw between two sensors; using an adjustable frequency between 50 to $200 \mathrm{~Hz}$.

Four sensors were fixed on the upper and lower limbs in two different sports, in order to capture inter-limb coordination: (i) in breaststroke swimming (cyclic activity), upper-lower coordination was analysed according to [2]: continuous relative phase (CRP) reveals flexionextension coupling between elbow (relative angle between forearm and arm) and knee (relative angle between leg and thigh) (Fig. 1); (ii) in rock climbing (discrete activity) on a artificial climbing wall, upper-lower coordination was analysed as regards yaw of hands (sensor fixed on the dorsal face of the hand) and yaw of foots (sensor fixed on the dorsal face of the foot). 


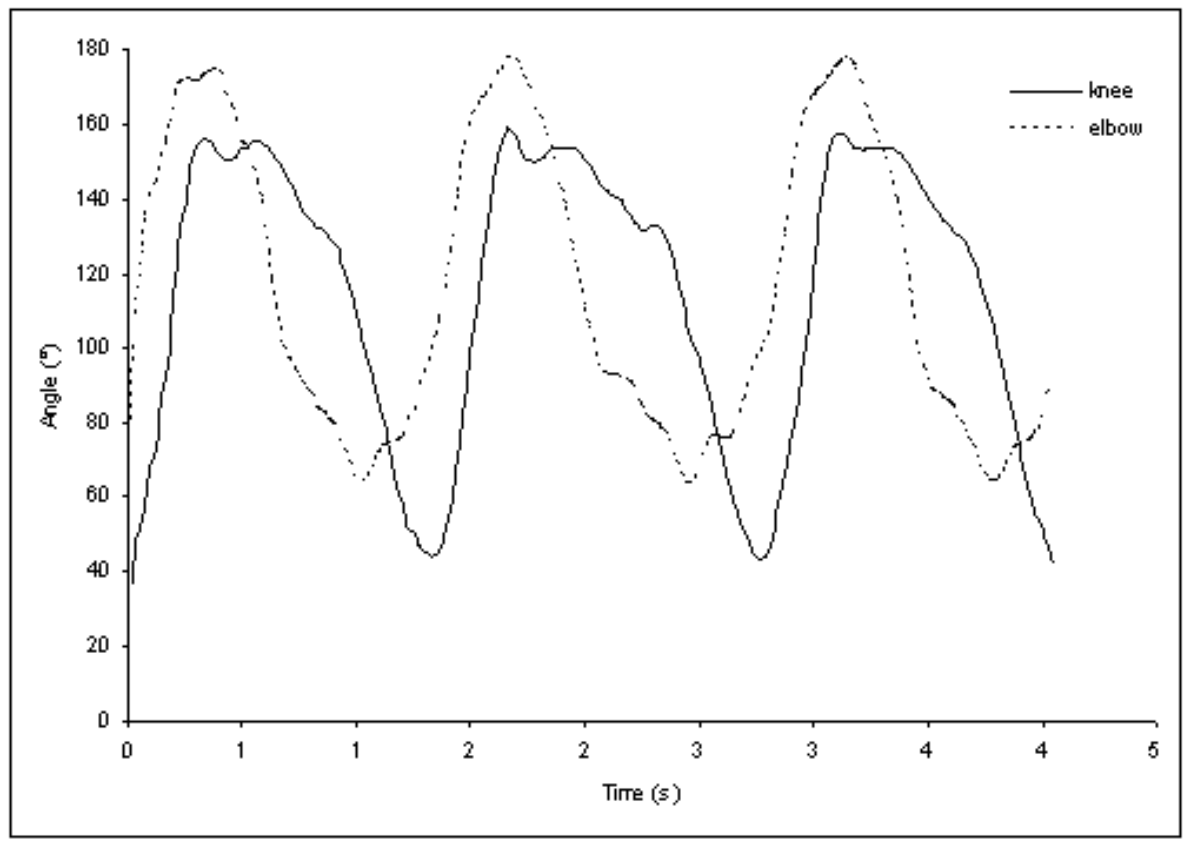

Figure 1. Knee and elbow angles time-series for non-expert breaststroke swimmer.

\section{Results}

Motor skill in terms of inter-limb coordination, corresponds to greater intra-individual variability, both for breaststroke swimming than rock climbing, suggesting that expert practitioners explore further modes of coordination. For example, in breaststroke

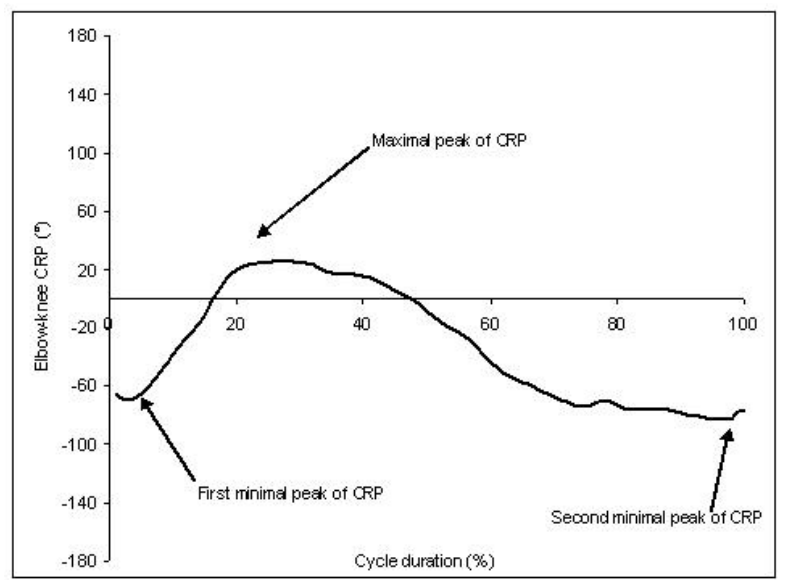

beginners showed lower elbow-knee CRP intracycle variation, supporting that elbow and knee angles mostly move in in-phase mode (like an accordion), while expert breaststrokers further varied their elbow-knee CRP due to alternation of in-phase and out-of-phase limb coupling (Fig. 2 and 3).

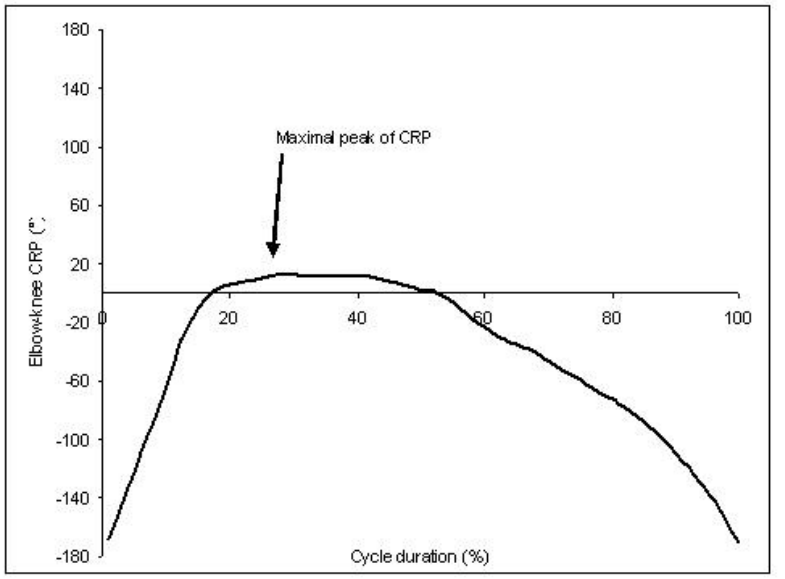

Figure 2. Knee-elbow continuous relative phase (CRP) for beginners (left) and experts (right) for 1 cycle taken in the time-serie. 

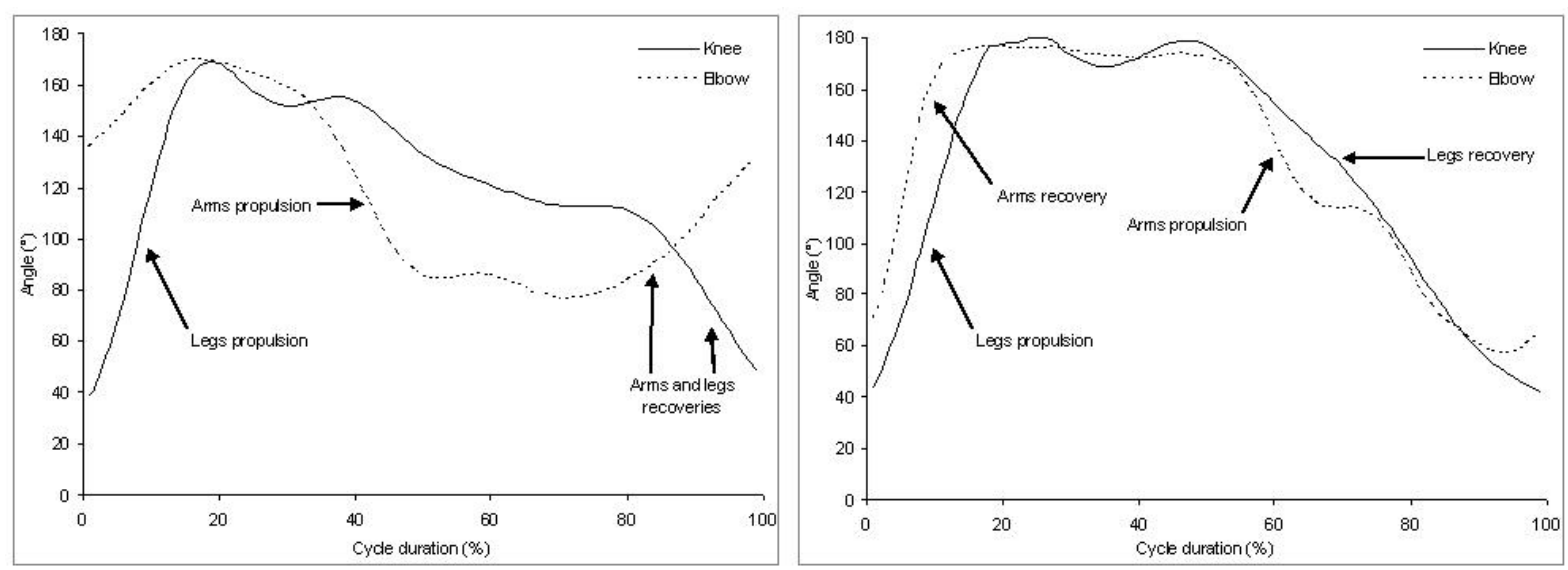

Figure 3. Knee and elbow angles for beginners (right) and experts (left) for 1 cycle taken in the time-serie.

In rock climbing, experts climbers exhibited alternation of in-phase and anti-phase modes of coordination, meaning that the foot yaw alternated between body face to the wall ("duck" foot position) and body in a profile position (one foot yaw on the right, while the other yaw foot is on the left) (Fig. 4). Conversely, beginners mostly used a "face" body position with foots in the duck position (Fig. 5).

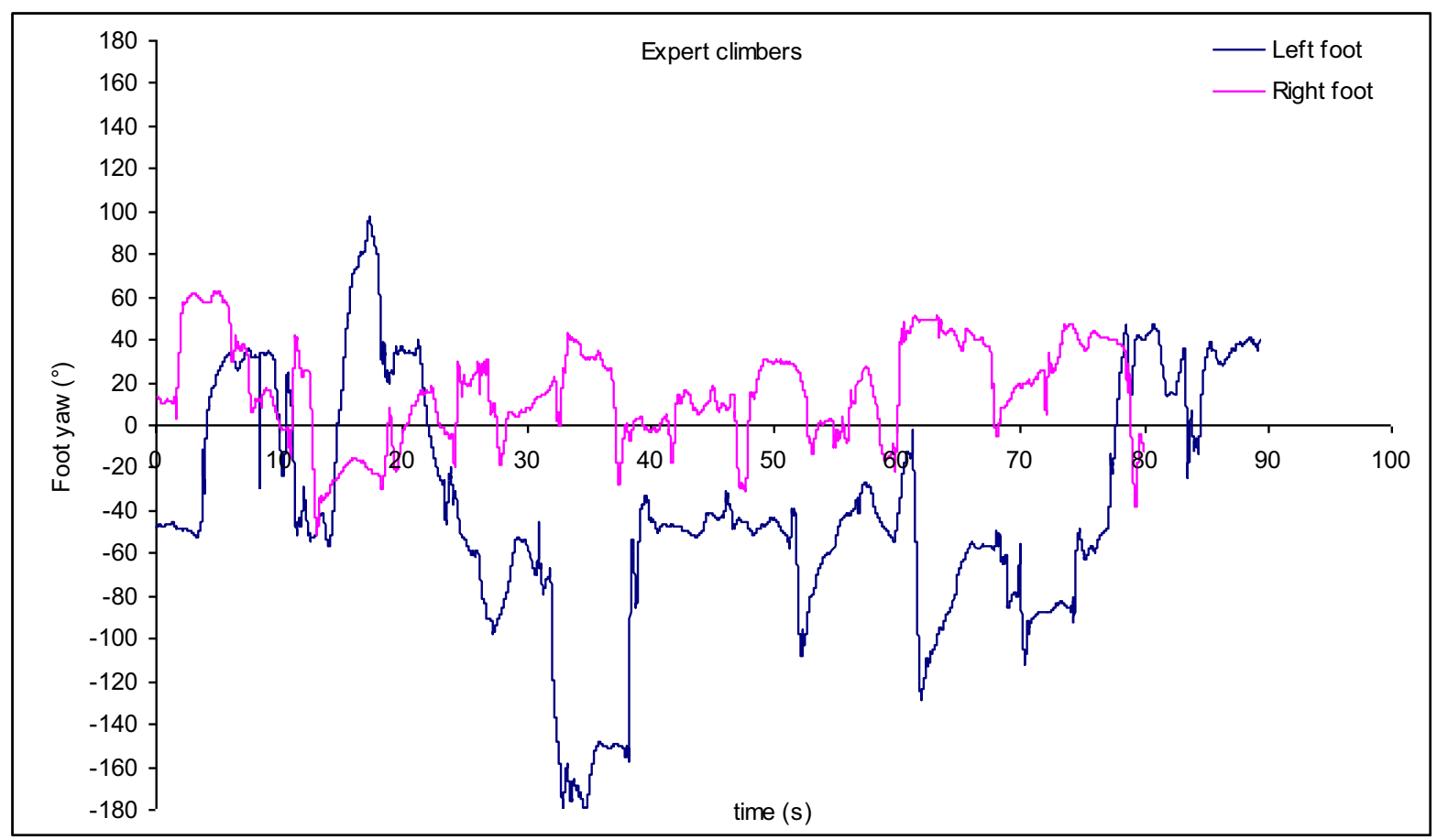

Figure 4. Yaw of left and right foot during an ascent of $10 \mathrm{~m}$ 


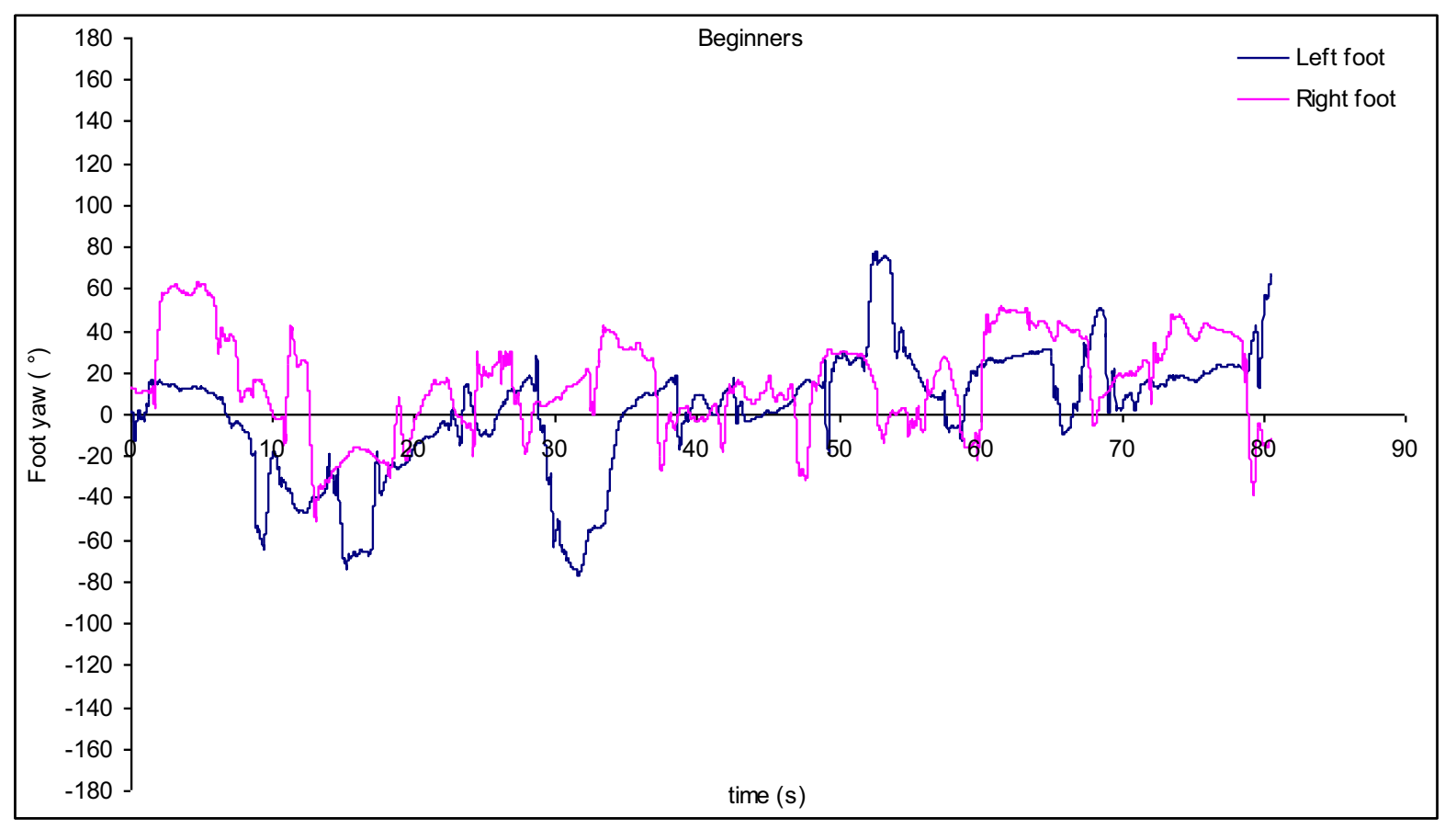

Figure 5. Yaw of left and right foot during an ascent of $10 \mathrm{~m}$.

\section{Discussion}

Newell [3] and Davids et al. [4] showed that intra-individual variability could have a functional role; notably individual adapted their motor behaviour to several interacting constraints (i.e. organismic, task, environmental). The use of inertial central enabled to analyse human behaviour by respecting the sociocultural constraints (e.g. environmental constraints that reflect the ecological context) and in the same time to provide long timeseries of data useful for intra-individual variability analysis.

Our results showed that the low variability of the coordination modes observed in beginners could be explained by their focus on the environmental constraints (e.g., control of buoyancy, gravity/Archimedes principle in swimming ; control of body equilibrium and gravity in rock climbing) while the high movement variability in experts could be explained by their capability to adapt to interacting constraints (e.g., adjust the glide time in swimming according to swim speed and active drag; alternation of face and profile body position in climbing according to movement, body equilibrium or rest focus) in order to get higher efficiency.
In summary, movement variability allows the performer to explore different motor solutions, facilitating the discovery of functional patterns of coordination and is supported by neurobiological degeneracy, i.e. "the ability of elements that are structurally different to perform the same function or yield the same output" (pp. 13763) [5].

\section{References}

[1] J.A.S. Kelso. Dynamics Patterns, MIT Press, 1995

[2] L. Seifert, H. Leblanc, D. Chollet and D. Delignières. Inter-limb coordination in swimming : effect of speed and skill level, Human Movement Science, 29, 103-113, 2010

[3] K.M Newell. Constraints on the development of coordination. In M.G. Wade \& H.T.A. Whiting (Eds.) Motor development in children: aspect of coordination and control, Dordrecht, Nijhoff, 341360, 1986

[4] K. Davids, S. Bennett, \& K. Newell. Movement system variability, Champaign, IL: Human Kinetics Publishers, 2006

[5] G.M. Edelman, J.A. Gally. Degeneracy and complexity in biological systems. Proc. Natl. Acad. Sci. U. S. A. 98, 24, 13763-13768, 2001 